\title{
Evaluation of Biomarkers for the Prediction of Venous Thromboembolism in Ambulatory Cancer Patients
}

\author{
Ruth Maria Schorling ${ }^{a} \quad$ Christian Pfrepper ${ }^{b}$ Thomas Golombek ${ }^{a}$ \\ Chiara Alessandra Cellac, d Nerea Muñoz-Unceta ${ }^{\mathrm{e}}$ Roland Siegemund ${ }^{\mathrm{b}}$ \\ Christoph Engel $^{f}$ Sirak Petros ${ }^{b}$ Florian Lordick $^{a}$ Maren Knödler ${ }^{a}$ \\ a University Cancer Center Leipzig (UCCL), University Hospital Leipzig, Leipzig, Germany; ${ }^{\mathrm{b}}$ Division of \\ Haemostaseology, University Hospital Leipzig, Leipzig, Germany; ' European Institute of Oncology, Milan, Italy; \\ ${ }^{\mathrm{d}}$ Department of Molecular and Translational Medicine, University of Brescia, Brescia, Italy; ${ }^{\mathrm{e}}$ Hospital Universitario \\ Marqués de Valdecilla, Santander, Spain; Institute for Medical Informatics, Statistics and Epidemiology, University \\ Leipzig, Leipzig, Germany
}

\section{Keywords}

Venous thromboembolism · Biomarkers · D-dimer ·

Risk assessment models · Cholangiocarcinoma

\begin{abstract}
Background: Venous thromboembolism (VTE) is a common complication of cancer. This study aimed to evaluate immature platelet fraction (IPF), mean platelet volume (MPV), Pselectin, D-dimer, and thrombin generation (TG) as predictive biomarkers for VTE and further the improvement of existing risk assessment models (RAMs). Methods: A prospective, observational, exploratory study was conducted on ambulatory cancer patients with indication for systemic chemotherapy. Baseline RAMs included the Khorana-, Vienna Cancer, Thrombosis-, Protecht-, ONKOTEV-, and Catscore. IPF, MPV, P-selectin, D-dimer, and TG were analysed at baseline and 3-month follow-up. Results: We enrolled 100 patients, of whom 89 completed the follow-up. Frequent tumour types were breast (30\%), gastric (14\%), gynaecological (14\%), and colorectal (14\%) cancer. Ten of the 89 patients (11.2\%) developed VTE. The highest VTE rate was observed in patients with cholangiocarcinoma $(3 / 5 ; 60 \%)$. Baseline Ddimer levels but not IPF, MPV, or P-selectin were associated with the risk of developing VTE (HR 6.9; $p=0.021)$. None of the RAMs showed statistical significance in predicting VTE. Peak thrombin and endogenous thrombin potential were
\end{abstract}

lower in patients who developed VTE. Biomarker changes between baseline and follow-up were not associated with VTE risk. Conclusions: VTE risk was well predicted by baseline D-dimer levels. Adding D-dimer could improve existing RAMs to better identify patients who may benefit from primary VTE prophylaxis. The VTE risk among patients with cholangiocarcinoma should be further evaluated.

(C) 2020 The Author(s)

Published by S. Karger AG, Basel

\section{Introduction}

Venous thromboembolism (VTE) is a frequent complication of cancer and one of the leading causes of death in cancer patients [1]. Compared to the general population, the risk of VTE is up to 7-fold higher in cancer patients [2]. The lack of robust data for evidence-based guidelines for prophylactic anticoagulation in ambulatory cancer patients results in low-grade recommendations for routine use of anticoagulant drugs [3]. Several approaches to stratify the VTE risk via risk assessment models (RAMs) have been proposed, including the Khorana [4], the Vienna Cancer and Thrombosis (CATS) [5], the Protecht [6], and the ONKOTEV scores [7], including

R.M.S. and C.P. contributed equally to this article. 
clinical and laboratory parameters. Most recently, the Catscore was established by Pabinger et al. [8] using Ddimer levels and tumour localization for VTE risk calculation.

Another focus of thrombosis research lies on quantitative and qualitative cellular abnormalities. While thrombocytosis has been established as an independent risk factor for VTE in several studies [7, 9], platelet-specific parameters such as immature platelet fraction (IPF) and mean platelet volume (MPV) have barely been studied in the context of cancer and VTE. However, first observations indicate an association of P-selectin, $\mathrm{D}$-dimer, and thrombin generation (TG) parameters with VTE risk $[10,11]$.

\section{Immature Platelet Fraction}

The fraction of reticulated platelets is called IPF and is a marker of platelet turnover. Reticulated platelets have a remaining protein synthesis capability [12] and tend to participate in arterial thrombus formation [13]. Higher levels of IPF have been measured in patients with major adverse cardiovascular events, including the acute coronary syndrome [14], cardioembolic stroke [15], and cardiovascular death [16]. While IPF has been investigated in myeloproliferative diseases [17], there are no published data on its possible correlation with the incidence of VTE in solid tumours.

\section{Mean Platelet Volume}

MPV is inversely correlated with platelet count [18] and is a parameter of platelet activity associated with higher platelet aggregation [19], increased expression of glycoprotein IIb-IIIa [20], and thromboxane B2 release [21]. While an association of increased MPV with thromboembolic diseases has been established in numerous studies in non-cancer patients [22-25], the role of MPV in patients with cancer and VTE remains unclear. CATS showed that MPV was significantly lower in cancer patients than healthy individuals, but the difference was minimal (10.2 vs. $10.3 \mathrm{fL}, p=0.022$ ) [26]. In that study, MPV levels above the 75th percentile were associated with a lower VTE risk, lower all-cause mortality, and a better prognosis of cancer.

\section{P-Selectin and D-Dimer}

P-selectin is expressed on platelets and endothelial cells and mediates cell interaction. In CATS, P-selectin levels were significantly higher in patients who developed VTE. The risk of VTE was 2.5-fold higher for patients with P-selectin levels above the 75th percentile, corresponding to $53 \mathrm{ng} / \mathrm{mL}$ [10]. D-dimers are a product of fibrinolysis routinely used to exclude VTE because of their high negative predictive value. In the CATS cohort, baseline $\mathrm{D}$-dimers were significantly higher in patients who developed VTE during the observation period [11]. The risk of VTE in patients with D-dimer levels above the 75th percentile was significantly elevated (hazard ratio, HR, 1.8, 95\% CI 1.0-3.2).

\section{Thrombin Generation}

TG is a functional assay determining the global coagulation capacity. Several studies have shown that TG is increased in patients with cancer [27, 28]. While patients with an elevated peak thrombin had a higher risk of developing VTE in CATS [29], another recent study from the same group of investigators failed to detect an association between TG parameters and the development of VTE. Interestingly, peak thrombin decreased during chemotherapy and the course of the disease [30].

The aim of this study was to evaluate IPF, MPV, Pselectin, D-dimer, and TG parameters at 2 time points as potential biomarkers for the prediction of VTE in patients with cancer. Five established risk assessment scores were applied at baseline and compared regarding their predictive value.

\section{Methods}

\section{Study Design and Population}

This study was conducted within the framework of the ONKOTEV 2 study, which is a prospective, observational multicentre study validating the previously established ONKOTEV score for VTE risk assessment. The present study comprises a part of the ONKOTEV 2 study population recruited at the University Cancer Center Leipzig (UCCL) between August 2016 and March 2017. It was conducted as a primarily exploratory evaluation of biomarkers independently from the ONKOTEV 2 analysis. The study was approved by the Ethics Committee of the University of Leipzig and conducted in accordance with the Declaration of Helsinki. Study inclusion criteria were as follows: (1) newly diagnosed or recurred cancer of the breast, gastrointestinal tract, prostate, gynaecological system, urinary tract, lung, head, and neck; (2) histologically confirmed diagnosis; (3) planned treatment with chemotherapy including targeted drugs; (4) written informed consent; and (5) age $\geq 18$ years. Exclusion criteria were chemotherapy within the previous 3 months, surgery within the previous 2 weeks, ongoing treatment with anticoagulants other than acetylsalicylic acid, or evidence of VTE at study inclusion. Information concerning the history of VTE was assessed in an individual interview. Blood samples were drawn before the start of anti-cancer treatment marking the beginning of the 3-month observation period. The follow-up investigation after 3 months included another blood sampling and evaluation of VTE incidents including the review of available imaging for staging purposes.

\section{Risk Assessment Models}

Baseline data collection and laboratory tests included the risk assessment according to Khorana score, Vienna CATS score, Protecht score, ONKOTEV score, and Catscore. The Khorana score stratifies into low-risk (0-1 patient), intermediate-risk ( 2 patients), and high-risk ( $\geq 3$ patients) groups by assigning 2 points to veryhigh-risk cancer sites (pancreatic or gastric), 1 point to high-risk cancer sites (lung, ovary, or bladder) and 1 point each for platelet 


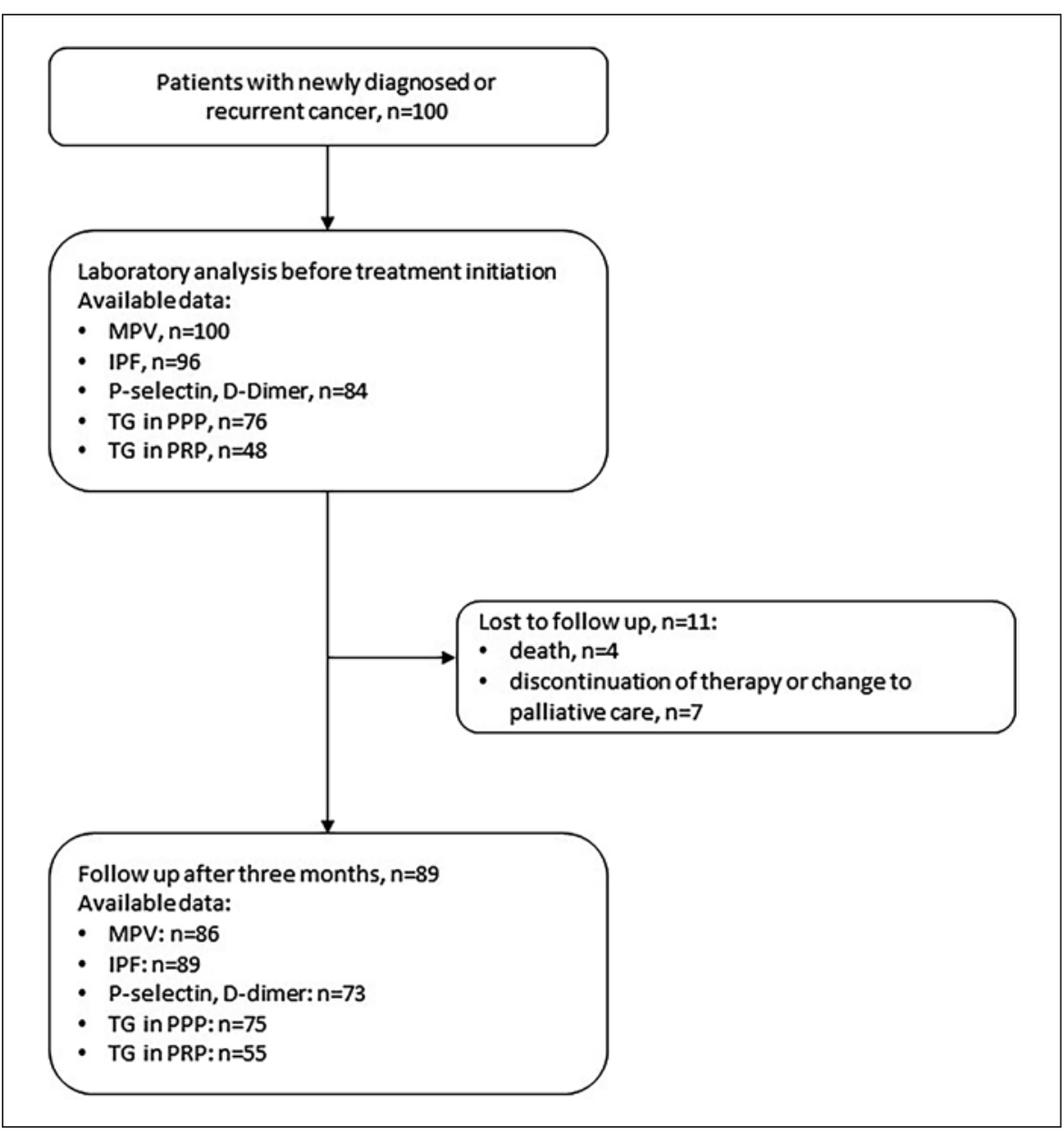

Fig. 1. Study design and data availability.

count $\geq 350 \times 10^{9} / \mathrm{L}$, haemoglobin $<10 \mathrm{~g} / \mathrm{dL}$, and/or use of erythropoietin-stimulating agents, leukocyte count $>11 \times 10^{9} / \mathrm{L}$, and body mass index $\geq 35 \mathrm{~kg} / \mathrm{m}^{2}$. The Vienna CATS group enhanced the Khorana risk score with the inclusion of 2 additional blood biomarkers: D-dimer and soluble P-selectin. The Protecht score is an expansion of the Khorana score, adding platinum- and gemcitabine-based chemotherapy as predictive variables, while the ONKOTEV score includes further clinical parameters such as metastatic disease, vascular or lymphatic compression, and a history of VTE. The Catscore represents a simplified risk model including only tumour site and D-dimer level, calculating the absolute 6-month VTE risk and suggesting a threshold at $10 \%$ for stratification into low- and high-risk patients [8]. The grouping of high- and low-risk tumour entities applied in the Catscore differ from the established classification in the Khorana score [8].

\section{Blood Sampling and Laboratory Analysis}

Blood samples were collected in vacuum containers with ethylenediamine tetraacetic acid (EDTA) or citrate. MPV and IPF were measured within $2 \mathrm{~h}$ after venipuncture with a Sysmex $\mathrm{XN}-9000^{\mathrm{TM}}$ haematology analyser. Soluble P-selectin levels were assessed in a human sP-selectin immunoassay (R\&D Systems ${ }^{\circledR}$, Minneapolis, MN, USA) using an Apollo 11 ELISA photometer, while D-dimer levels were determined by the particle-enhanced immunoturbidimetric assay INNOVANCE ${ }^{\circledR}$ D-DIMER (Siemens Medical Solutions) on the Behring coagulation system analyser. TG was assessed using the calibrated automated thrombogram and commercially available test kits according to the manufacturer's instructions on a Fluoroskan Ascent (ThermoLabsystems OY,
Helsinki, Finland) at 360/460 nm wavelength. Blood samples were centrifuged at $18^{\circ} \mathrm{C}$ and $1,000 \mathrm{rpm} 170 \mathrm{~g}$ for $10 \mathrm{~min}$ to prepare platelet-rich plasma (PRP), and then at another 4,000 rpm 1,800 g for $20 \mathrm{~min}$ to prepare platelet-poor plasma (PPP). Calibrated automated thrombogram was carried out using $5 \mathrm{pm}$ tissue factor as a final concentration. TG parameter lag time, endogenous thrombin potential (ETP), peak thrombin, and time to peak (TTP) were measured. In case of blood sampling from an implanted port system, residual heparin levels were measured and antagonised using polybrene [31] before the TG assay was performed.

\section{Diagnosis of VTE}

Regular screenings for VTE were not performed but duplex ultrasound and/or computer tomography were carried out in case of clinical symptoms. Incidental, asymptomatic VTE was counted as an event if anticoagulant treatment was required. VTE was defined as deep vein thrombosis (DVT) at any location including limb and abdomen as well as pulmonary embolism (PE).

\section{Statistical Analyses}

Descriptive data are presented using medians and interquartile ranges (IQR) for continuous variables and absolute numbers and percentages for categorial variables. Standard nonparametric tests were run for hypothesis testing: the Kruskal-Wallis test was applied to test for differences between various tumour sites, whereas the Mann-Whitney U test was used to analyse differences in continuous over dichotomous variable distribution. Correlations between continuous variables are described by Spearman's coefficient. We evaluated whether RAMs, IPF, MPV, P-selectin, D-di- 
Table 1. Baseline characteristics

\begin{tabular}{|c|c|c|}
\hline Variable & $\begin{array}{l}\text { All cancer } \\
\text { patients }(n=100)\end{array}$ & $\begin{array}{l}\text { VTE during } \\
\text { follow-up }(n=10)\end{array}$ \\
\hline Median age (IQR), years & $58(51-68)$ & $62(56-72)$ \\
\hline \multicolumn{3}{|l|}{ Gender, $n$} \\
\hline Female & 62 & 6 \\
\hline Male & 38 & 4 \\
\hline \multicolumn{3}{|l|}{ Tumour site grouped by risk category, $n$ (at follow-up) } \\
\hline Breast & $30(29)$ & 2 \\
\hline Stomach/gastro-oesophageal junction & $14(13)$ & 1 \\
\hline Pancreas & $6(4)$ & 0 \\
\hline Colorectal & $14(14)$ & 2 \\
\hline Lung & $4(2)$ & 1 \\
\hline Genitourinary tract & $16(13)$ & 1 \\
\hline Cholangiocarcinoma & $6(5)$ & 3 \\
\hline $\begin{array}{l}\text { Other (head and neck, oesophagus, prostate, cancer of } \\
\text { unknown primary) }\end{array}$ & $10(9)$ & 0 \\
\hline \multicolumn{3}{|l|}{ Tumour stage, $n$} \\
\hline Localized disease & 36 & 1 \\
\hline Distant metastases & 64 & 9 \\
\hline First diagnosis, $n$ & 82 & 10 \\
\hline Personal history of VTE, $n$ & 6 & 1 \\
\hline Vascular/lymphatic macroscopic compression & 5 & 1 \\
\hline Body mass index (IQR), $\mathrm{kg} / \mathrm{m}^{2}$ & $24(22-28)$ & $23(21-25)$ \\
\hline \multicolumn{3}{|l|}{ Laboratory parameters (IQR) } \\
\hline Platelet count, $\times 10^{9} / \mathrm{L}$ & $298(242-374)$ & $330(216-409)$ \\
\hline Leucocyte count, $\times 10^{9} / \mathrm{L}$ & $7.9(3.1-9.7)$ & $8.5(6.4-10.9)$ \\
\hline Haemoglobin, g/dL & $12.7(11.2-13.9)$ & $12.0(10.3-13.5)$ \\
\hline Mean platelet volume, $\mathrm{fL}$ & $10.0(9.3-10.7)$ & $9.7(9.2-11.0)$ \\
\hline Immature platelet fraction, $\%$ & $2.8(2.0-4.3)$ & $2.3(1.9-4.9)$ \\
\hline $\mathrm{D}$-dimer, $\mu \mathrm{g} / \mathrm{mL}$ & $1.2(0.6-2.1)$ & $2.8(1.5-4.6)$ \\
\hline Soluble P-selectin, ng/mL & $47.0(37.0-63.0)$ & $49(34.8-83.5)$ \\
\hline Lag time in PPP, min & $2.2(1.5-3.0)$ & $3.2(1.8-5.8)$ \\
\hline Time to peak thrombin in PPP, min & $4.5(3.7-5.7)$ & $6.7(4.1-10.4)$ \\
\hline Peak thrombin in PPP, nM & $364(275-447)$ & $260(113-328)$ \\
\hline Endogenous thrombin potential in $\mathrm{PPP}, \mathrm{nM} \times \min$ & $1,917(1,659-2,354)$ & $1,689(1,240-1,860)$ \\
\hline Lag time in PRP, min & $8.8(6.9-11.0)$ & $10(6.7-16.8)$ \\
\hline Time to thrombin peak in PRP, min & $15.7(12.9-20.1)$ & $16.0(13.7-25.2)$ \\
\hline Peak thrombin in PRP, nM & $194(164-251)$ & $186(130-214)$ \\
\hline Endogenous thrombin potential in $\mathrm{PRP}, \mathrm{nM} \times \min$ & $2,308(1,945-2,978)$ & $1,943(1,705-2,533)$ \\
\hline Median observation time (IQR), days & $92(87-100)$ & \\
\hline \multicolumn{3}{|l|}{ Cancer treatment during the observation period, $n$} \\
\hline Cisplatin based & 13 & 3 \\
\hline Other platinum-based compounds & 51 & 6 \\
\hline Non-platinum-based chemotherapy & 36 & 1 \\
\hline
\end{tabular}

Laboratory parameters are given as medians unless indicated otherwise.

mer, or TG parameters at baseline were predictive of VTE. Risk scores were dichotomized into low- and high-risk groups as predefined in the original literature, while biomarkers were evaluated dichotomously using the 75th percentile as the cut-off. Using the competing risk regression model by Fine and Gray [32], the predictive value was evaluated by estimating the sub-distribution $H R$. Death was treated as competing event for VTE. Cumulative incidences with 95\% CIs were calculated in a competing risk analysis using Choudhury's method [33]. Statistical analyses were performed using SPSS version 22. The competing risk analysis was assessed in R, version 3.5.0 (R Foundation for Statistical Computing, Vienna, Austria), using the cmprsk package version 2.2-7.

\section{Results}

\section{Study Population}

We enrolled 100 patients between August 2016 and March 2017, of whom 89 patients completed the followup procedures after a median of 92 (IQR 87-100) days. Overall, 4 patients died, and 7 patients dropped out due to premature discontinuation of systemic therapy (Fig. 1). Baseline characteristics of the study population are shown in Table 1. 
A

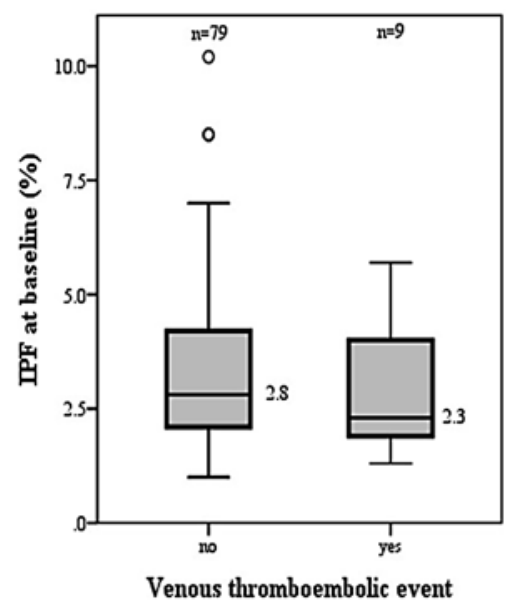

B

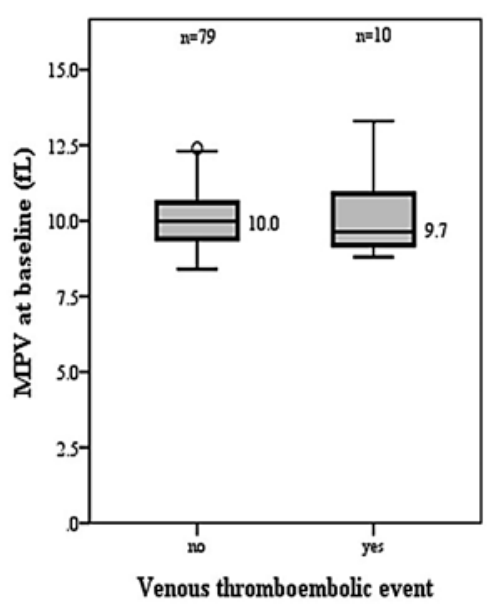

$\mathrm{C}$

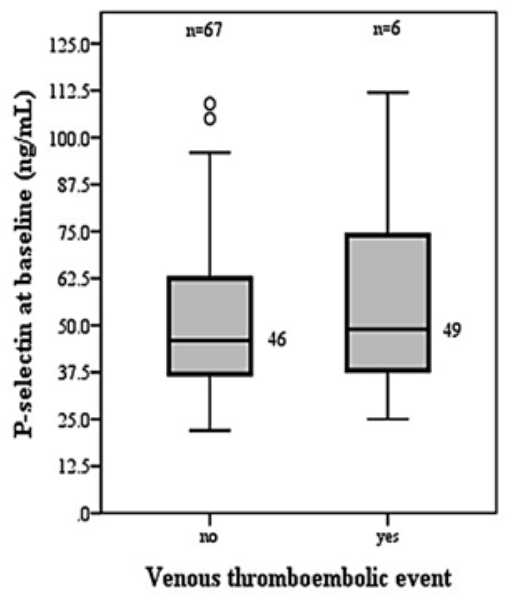

Fig. 2. IPF (A), MPV (B), and P-selectin (C) levels at baseline, according to the occurrence of a venous thromboembolic event during 3 months of follow-up $(p=0.815 ; p=0.633$; and $p=0.680$, respectively).

Table 2. Study population and venous thromboembolism (VTE) rates according to risk assessment models

\begin{tabular}{|c|c|c|c|c|}
\hline Risk assessment model & $\begin{array}{l}\text { Total study } \\
\text { population }(n=100)\end{array}$ & $\begin{array}{l}\text { VTE during } \\
\text { follow-up }(n=10)\end{array}$ & $\begin{array}{l}\text { HRs for VTE } \\
\text { occurrence }(95 \% \mathrm{CI})\end{array}$ & $p$ value \\
\hline \multicolumn{5}{|l|}{ Khorana score $(n=100), n(\%)$} \\
\hline Low-risk group (0 patient) & $32 / 100$ & $5 / 30(16.7)$ & & \\
\hline Intermediate-risk group ( $1-2$ patients) & $57 / 100$ & $4 / 53(7.5)$ & & \\
\hline High-risk group ( $\geq 3$ patients) & $11 / 100$ & $1 / 9(11.1)$ & $0.9(0.1-7.5)$ & 0.951 \\
\hline \multicolumn{5}{|l|}{ ONKOTEV score $(n=100), n(\%)$} \\
\hline $0-1$ patients & $83 / 100$ & $7 / 78(9.0)$ & & \\
\hline$\geq 2$ patients & $17 / 100$ & $3 / 14(21.4)$ & $2.5(0.7-9.1)$ & 0.182 \\
\hline \multicolumn{5}{|c|}{ Vienna Cancer and Thrombosis score $(n=72), n(\%)$} \\
\hline $0-2$ patients & $43 / 72(59.7)$ & $3 / 43(7.0)$ & & \\
\hline$\geq 3$ patient & $29 / 72(40.3)$ & $3 / 29(10.3)$ & $1.5(0.3-7.2)$ & 0.621 \\
\hline \multicolumn{5}{|l|}{ Protecht score $(n=100), n(\%)$} \\
\hline $0-2$ patients & $73 / 100$ & $7 / 69(10.1)$ & & \\
\hline$\geq 3$ patients & $27 / 100$ & $3 / 23(13.4)$ & $1.3(0.3-4.9)$ & 0.720 \\
\hline Catscore $(n=84), n(\%)$ & 6.38 & 6.84 & $0.9(0.1-7.3)$ & 0.891 \\
\hline$<10 \%$ & $71 / 84(84.5)$ & $6 / 66(9.1)$ & & \\
\hline$\geq 10 \%$ & $13 / 84(15.5)$ & $1 / 13(7.7)$ & & \\
\hline
\end{tabular}

\section{Occurrence of VTE}

Ten of the 89 patients (11.2\%) developed VTE during the 3 -month follow-up period, including 5 isolated DVTs, 3 isolated PEs, 1 combined DVT plus PE, and 1 portal vein thrombosis. Median time from study inclusion to VTE was 27 days (22-59). VTE rates according to the risk assessment scores and HR for high-risk score groups are listed in Table 2.

Nine out of 10 VTEs occurred in patients with platinbased chemotherapy: in the group of patients receiving cisplatin, carboplatin, and oxaliplatin, 25.0\% (3/12), $14.3 \%(3 / 21)$, and $12.0 \%(3 / 25)$ developed VTE, respectively. Only 1 of 31 (3.2\%) patients with non-platin-based chemotherapy developed VTE.
The highest VTE rate was observed in the group of cholangiocarcinoma patients of whom $3 / 5$ (60\%) with completed follow-up data developed VTE (2 DVTs and $1 \mathrm{PE})$. The VTE distribution by tumour site in patients with completed follow-up data was: lung cancer $1 / 2$ (50\%), colorectal cancer $2 / 14(14.3 \%)$, gastric cancer $1 / 13(7.7 \%)$, breast cancer $2 / 29(6.9 \%)$, and genitourinary cancer $1 / 13(7.7 \%)$. No patient with pancreatic cancer and completed follow-up (0/4) developed VTE. All patients with cholangiocarcinoma had platinum-based chemotherapy, and all but 1 patient had metastatic disease. 


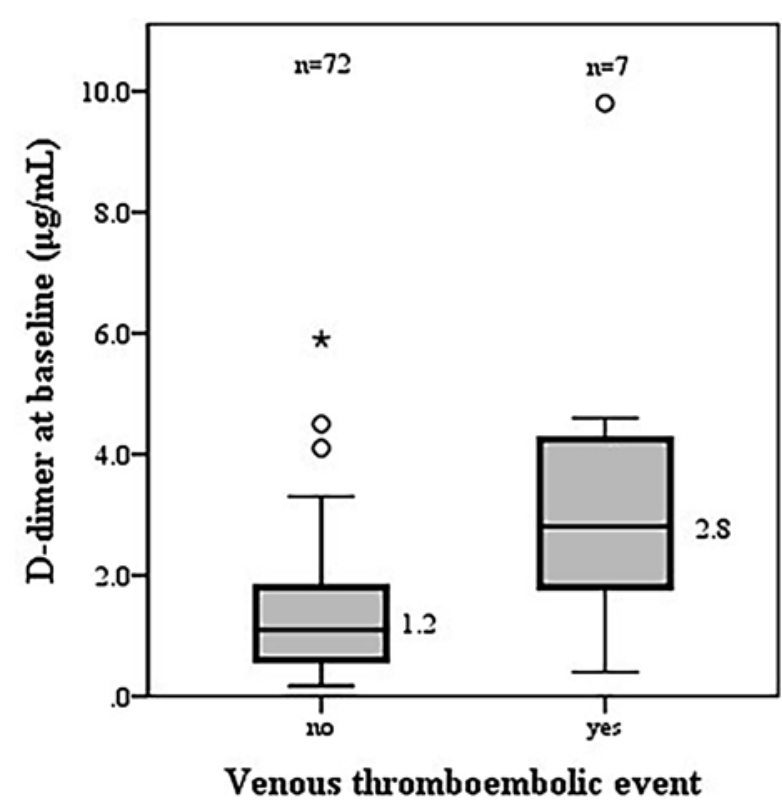

Fig. 3. D-dimer levels according to the occurrence of a venous thromboembolic event during 3 months of follow-up $(p=0.031)$.

Biomarkers at Baseline and Their Association with VTE MPV, IPF, and P-Selectin

Baseline IPF was available in 96 patients and showed a median level of 2.8\% (IQR 2.0-4.3). MPV was assessed in all patients at study entry (median 10.0 fL, IQR 9.3-10.7). $\mathrm{P}$-selectin was available in 84 patients with a median level of $47 \mathrm{ng} / \mathrm{mL}$ (IQR 37-63). No association was found between levels of IPF, MPV, or P-selectin and the occurrence of VTE. There was no statistically significant difference in IPF, MPV, or P-selectin levels between the groups of patients with and without VTE $(p=0.815 ; p=0.633$; $p=0.680$, respectively), as shown in Figure 2.

A strong correlation between IPF and MPV was observed at study entry (Spearman coefficient: $r=0.839$, $p<0.001$ ), and both parameters showed a moderately significant negative correlation with platelet count (Spearman coefficient: $r=-0.364, p<0.001$ for IPF; $r=-0.420$; $p<0.001$ for MPV).

The distribution of IPF levels among various tumour types was significantly different (Table 3 ).

The highest median IPF level was seen in breast and the lowest in colorectal cancer. The distribution of MPV showed some differences among tumour sites without reaching statistical significance $(p=0.082)$. There was a significantly higher level of IPF in patients with newly diagnosed cancer compared to those diagnosed with recurrent cancers ( 3.0 vs. $2.5 \%, p=0.018$ ). IPF and MPV were not associated with gender, age, or status of distant metastasis.
Table 3. Median levels of immature platelet fraction (IPF) and mean platelet volume (MPV) associated with primary tumour site

\begin{tabular}{llc}
\hline Primary tumour site & IPF, \% & MPV, fL \\
\hline Breast $(n=30)$ & 3.6 & 10.2 \\
Lung $(n=4)$ & 3.3 & 10.0 \\
Gastropancreatic $(n=20)$ & 2.9 & 10.3 \\
Gynaecological/urological $(n=14)$ & 2.4 & 9.5 \\
Colorectal $(n=13)$ & 2.0 & 9.4 \\
Other $(n=19)$ & 2.4 & 10.0 \\
$p$ value & 0.041 & 0.082
\end{tabular}

$p$ value (Kruskal-Wallis test) for the association of IPF/MPV with the primary tumour site in the total study population.

\section{D-Dimer}

$\mathrm{D}$-dimer levels were available in 84 patients. Median D-dimer level was $1.2 \mu \mathrm{g} / \mathrm{mL}$ (IQR 0.6-2.1). Baseline Ddimer levels were significantly higher in patients who developed VTE (median $2.8 \mu \mathrm{g} / \mathrm{mL}$ ) compared to patients without VTE (median $1.15 \mu \mathrm{g} / \mathrm{mL}, p=0.031$; Fig. 3). Among 22 patients with baseline D-dimer levels above the 75 th percentile $(2.1 \mu \mathrm{g} / \mathrm{mL}), 5$ developed VTE (cumulative incidence: $22.8,95 \%$ CI 8.0-42.0) compared to 2 out of 57 patients (cumulative incidence: 3.5\%, 95\% CI 0.610.8 ) with $\mathrm{D}$-dimer levels $\leq 75$ th percentile (HR 6.9; 95\% CI 1.3-35.6; $p=0.021$; Fig. 4).

\section{Thrombin Generation}

TG in PRP at baseline was available in 48 and PPP in 76 patients. Eight patients who developed VTE had a significantly lower baseline peak thrombin $(p=0.005)$ and ETP $(p=0.036)$ in PPP compared to those who did not develop VTE (Fig. 5). There was no difference in PRP in these patient groups. Patients with P-selectin $\geq 53.1 \mathrm{ng} /$ $\mathrm{mL}$ had a lower median TTP in PRP $(p=0.05$; Fig. 6). There was no significant correlation between TG parameters and D-dimer, P-selectin, IPF, or MPV values and no significant correlation between TG parameters and Khorana-, Vienna CATS, and Protecht-, ONKOTEV scores, or Catscore.

\section{Biomarkers during Follow-Up}

A total of 89 patients completed the follow-up period and returned for the second blood sampling after a median of 92 (IQR 87-100) days. IPF, MPV, P-selectin, Ddimer, TG in PPP, and TG in PRP at this time point were available in $86,89,73,73,75$, and 55 patients, respectively.

Median changes in IPF, MPV, P-selectin, and D-dimer levels were $0.8 \%$ (IQR 0.3-1.5), $0.4 \mathrm{fL}$ (IQR 0.2-0.7), 21 $\mathrm{ng} / \mathrm{mL}$ (IQR $8.0-34.5$ ), and $0.6 \mu \mathrm{g} / \mathrm{mL}$ (IQR 0.2-1.4), respectively. Changes from baseline to follow-up were not 
Fig. 4. Cumulative incidence of venous thromboembolism in subgroups by baseline $\mathrm{D}$-dimer levels below and above the 75th percentile.

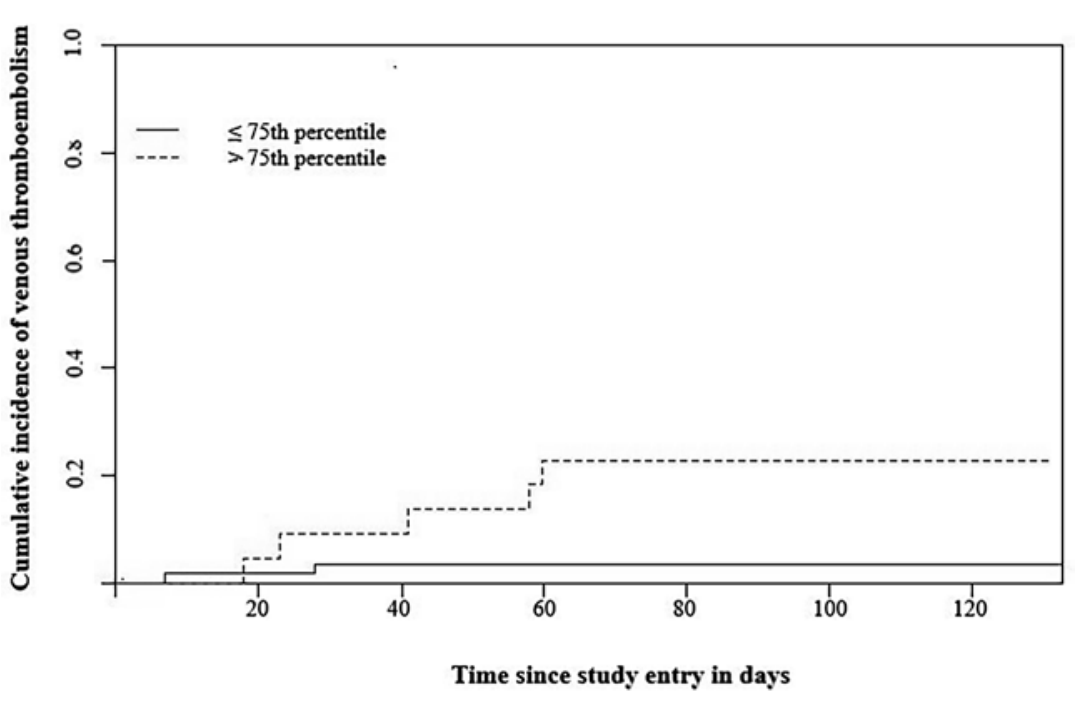

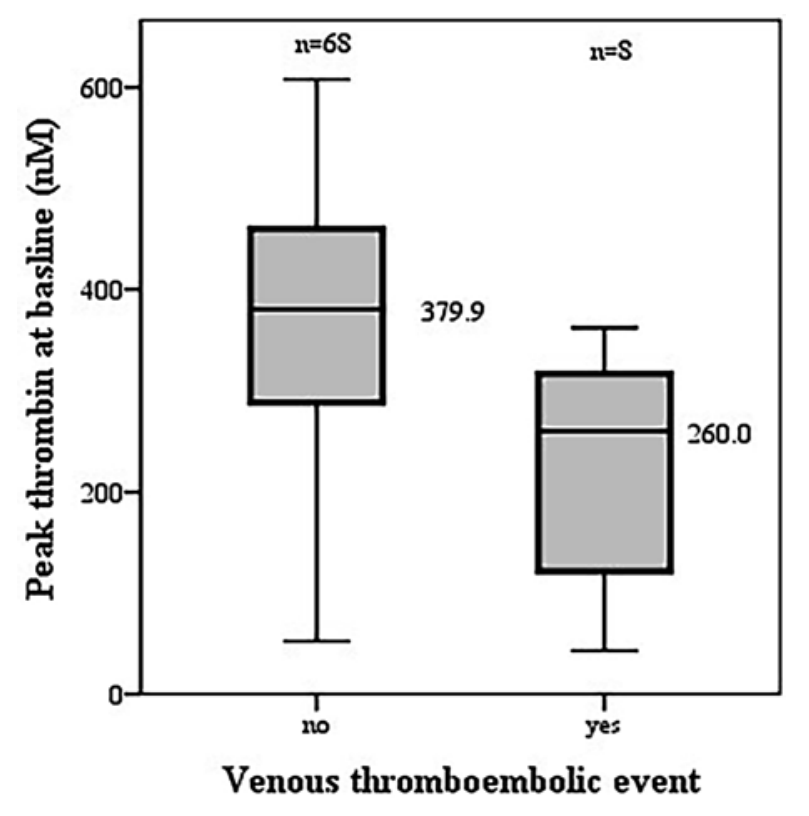

Fig. 5. Peak thrombin in platelet-poor plasma according to the occurrence of a venous thromboembolic event $(p=0.005)$.

significantly different between patients who did and did not develop VTE (changes in IPF $p=0.841$, MPV $p=$ 0.371 , P-selectin $p=0.968$, and D-dimer $p=0.951$ ). Regarding TG data, there was a trend towards a significant reduction in lag time between baseline and follow-up ( 2.17 vs. $1.67 \mathrm{~min}, p=0.054$ ), while TTP, ETP, and peak thrombin where not different between the 2 time points. TG parameters at follow-up were not significantly different whether patients developed VTE or not.

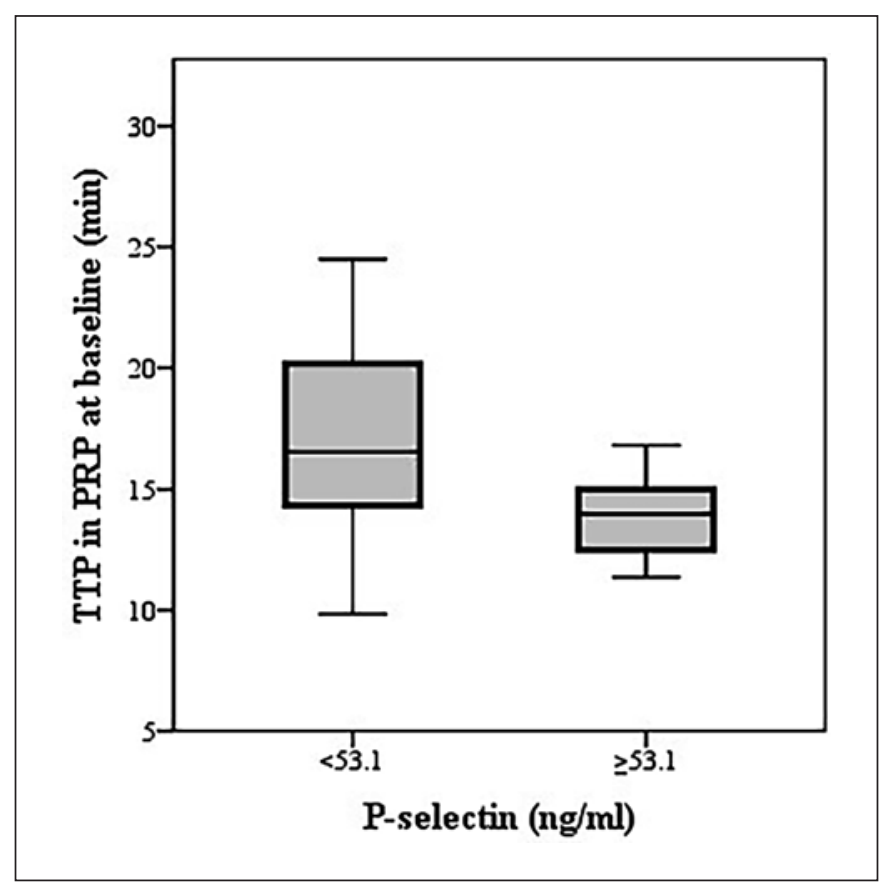

Fig. 6. Time to peak thrombin (TTP) in platelet-rich plasma (PRP) in patients with $\mathrm{P}$-selectin according to the 75th percentile in CATS $(<53.1 \mathrm{ng} / \mathrm{mL}[n=22]$ and $\geq 53.1 \mathrm{ng} / \mathrm{mL}[n=14], p=0.05)$.

\section{Discussion}

This study was particularly dedicated to explore several blood biomarkers (IPF, MPV, P-selectin, D-dimer, and TG parameters) to increase the accuracy of identifying ambulatory cancer patients at risk for VTE.

The most relevant finding is that $\mathrm{D}$-dimer levels before treatment initiation are predictive of VTE in cancer patients receiving ambulatory chemotherapy. Elevated D- 
dimer levels above the 75th percentile were associated with a more than 6-fold increased risk of VTE. This finding is in accordance with a previously published study from the Vienna group investigating the VTE risk in cancer patients [11]. In contrast, our study could not demonstrate a correlation between IPF, MPV, or P-selectin levels and the occurrence of VTE. These results do not confirm observations from CATS concerning the potential role of MPV and P-selectin. Riedl et al. [26] reported a significant decrease in VTE risk in patients with MPV levels above the 75th percentile. A possible explanation for this discrepancy could be the difference in patient characteristics at baseline and the incidence of VTE among different tumour groups. Regarding different tumour types, a significantly elevated VTE risk in patients with low MPV levels in CATS was described in the subgroup of pancreatic cancer patients after 6 months. In contrast, no pancreatic cancer patient developed VTE in our cohort, but follow-up might have been too short to detect the effect of MPV levels in our patients. MPV results should be handled carefully because MPV is easily influenced by technical and individual factors such as intake of anticoagulants and EDTA-containing tubes, which are known to cause platelet swelling in the course of time [34]. We used the same haematology analyser for all samples, defined use of anticoagulant drugs as exclusion criterion, and established a time shorter than $2 \mathrm{~h}$ from venipuncture to blood testing to anticipate and minimize these limitations.

Peak thrombin and ETP measured in PPP at baseline were lower in patients who developed VTE. Taking into account that these TG parameters were higher in patients developing thrombosis in the study by Ay et al. [29], while they were not different in the study from Reitter et al. [30], it seems that TG is not a good predictive parameter in cancer patients. This might be due to the high inter- and intra-individual differences and the dynamics of TG during the course of disease, so that a single measurement may not be appropriate. The $\mathrm{D}$-dimer level seems to better predict VTE in cancer patients than TG as it is a marker for the activation of the coagulation cascade and fibrin turnover, while TG only reflects the patients' potential to generate thrombin that is further modulated in vivo by external factors like endothelial and platelet activation by the tumour. This is in accordance with the finding that TTP measured in PRP was lower in patients with elevated $P$-selectin because in PRP the additional effect of platelets on TG is included.

Being aware of the relatively small study population, comparing the predictive value of different RAMs was not a primary objective of this study. Therefore, our evaluation is only exploratory. However, ONKOTEV scores showed the highest HR in the high-risk group but without reaching statistical significance. This could be explained by the high number of patients with distant metastases in our cohort, which is part of the ONKOTEV score. Distant metastases were recorded in $64 \%$ of the entire study population and in $90 \%$ of the patients who developed VTE. In contrast, only $39 \%$ of all patients showed distant metastasis in the CATS cohort.

The Catscore failed to predict VTE risk in our cohort despite the integration of D-dimer levels although the distribution of tumour sites in our cohort was comparable to the CATS cohort. However, only 1 VTE occurred in our very-high-risk group (5.6\%), and 15.9\% VTE occurred in our high-risk group when calculated according to the Catscore. The VTE rate in our high-risk group was mainly due to the high proportion of patients with cholangiocarcinoma which are not mentioned as a specific tumour entity in any existing RAM-defining study. There is only one retrospective study reporting a $14.7 \%$ VTE incidence in patients with cholangiocarcinoma [35]. Risk factors for the development of VTE in these patients were elevated C-reactive protein, metastatic disease, and chemotherapy. In comparison, the VTE incidence in patients with pancreas carcinoma was $14.9 \%$ in the Vienna CATS study [5] and $8.1 \%$ in the cohort published by Khorana et al. [36]. We strongly recommend further research on the VTE risk of cholangiocarcinoma patients and integration into existing RAMs, since our findings can only be regarded as exploratory due to small patient numbers. Assigning this entity to the very-high-risk tumour group improved the risk calculation in nearly all considered RAMs (online suppl. Table 1, for all online suppl. material see www.karger.com/doi/10.1159/000508271).

Nine out of 10 VTEs occurred in patients with platinum-based therapy without reaching a level of significance due to a high proportion of platinum-based regimes in our total cohort. However, the observed trend is in accordance with previous findings, showing a significantly elevated risk of VTE under cisplatin-based chemotherapy [37, 38].

There was a significant difference in IPF levels among different tumour types, with IPF levels being highest in breast cancer and lowest in colorectal cancer. The clinical relevance of this finding remains unclear, as both tumour types are not known as especially high or low VTE risk tumours. Furthermore, a strong correlation between IPF and MPV and a moderate negative correlation between platelet count, MPV, and IPF were observed at study entry, which would be physiologically expected. The higher MPV in patients with higher IPF might be explained by the fact that IPF represents immature platelets having a higher volume because they were recently shed from megakaryocytes. The negative correlation between IPF and platelet count is most likely caused by a higher platelet turnover in these patients. The significantly higher median IPF levels in patients with initial diagnosis com- 
pared to those diagnosed with progressive disease were also remarkable, which potentially indicating a stronger bone marrow reserve in patients without previous treatment.

The strength of the present study is the prospective study design and the use of well-known risk scores for screening. However, the results must be interpreted within the limitations of this study. One limitation of the study is the relatively small sample size and the diversity of the study population, especially regarding tumour type and stage, and the chemotherapy regimen. One might argue that diversity in tumour type and treatment modalities is part of the ambulatory reality, for which RAMs were designed. However, tumour types differ significantly regarding baseline VTE risk, which renders a comparison of different study populations difficult. Therefore, our results should be regarded as exploratory and require further prospective validation in specific patient cohorts. Further analyses in larger patient samples are necessary to clarify the predictive value of IPF, MPV, and P-selectin regarding the development of VTE in cancer patients.

\section{Conclusions}

VTE risk was well predicted by D-dimer baseline blood levels in our cohort. Adding D-dimer could improve existing RAMs to better identify high-risk patients who may benefit from primary VTE prophylaxis as realized in the Vienna CATS score and the Catscore. Further research is needed concerning the VTE risk in cholangiocarcinoma patients.

\section{Acknowledgements}

We thank all patients for contributing to the study.

\section{Statement of Ethics}

The ethical approval for the study was received from the Ethics Committee of the University of Leipzig in accordance with the Declaration of Helsinki. All participants gave written, informed consent for participation.

\section{Disclosure Statement}

The authors have no conflicts of interest to declare.

\section{Funding Sources}

Not applicable.

\section{Author Contributions}

R.M.S., C.P., C.A.C., S.P., F.L., and M.K. contributed to the study design. R.M.S., C.P., T.G., N.M.-U., and M.K. contributed to recruiting and data collection. R.S. contributed to laboratory testing. R.M.S., C.P., C.E., and M.K. contributed to the data analysis. R.M.S., C.P., T.G., C.A.C., N.M.-U., S.P., R.S., C.E., F.L., and M.K. contributed to the discussion. R.M.S., C.P., and M.K. contributed to the writing of the manuscript. All authors read and approved the final version of the manuscript.

\section{References}

1 Khorana AA, Francis CW, Culakova E, Kuderer NM, Lyman GH. Thromboembolism is a leading cause of death in cancer patients receiving outpatient chemotherapy. J Thromb Haemost. 2007 Mar;5(3):632-4.

2 Timp JF, Braekkan SK, Versteeg HH, Cannegieter SC. Epidemiology of cancer-associated venous thrombosis. Blood. 2013 Sep; 122(10):1712-23.

3 Farge D, Bounameaux H, Brenner B, Cajfinger F, Debourdeau P, Khorana AA, et al. International clinical practice guidelines including guidance for direct oral anticoagulants in the treatment and prophylaxis of venous thromboembolism in patients with cancer. Lancet Oncol. 2016 Oct;17(10):e452-66.

4 Khorana AA, Kuderer NM, Culakova E, Lyman GH, Francis CW. Development and validation of a predictive model for chemotherapy-associated thrombosis. Blood. 2008 May; 111(10):4902-7.

5 Ay C, Dunkler D, Marosi C, Chiriac AL, Vormittag R, Simanek R, et al. Prediction of venous thromboembolism in cancer patients. Blood. 2010 Dec;116(24):5377-82.
6 Verso M, Agnelli G, Barni S, Gasparini G, LaBianca R. A modified Khorana risk assessment score for venous thromboembolism in cancer patients receiving chemotherapy: the Protecht score. Intern Emerg Med. 2012 Jun; 7(3):291-2.

7 Cella CA, Di Minno G, Carlomagno C, Arcopinto M, Cerbone AM, Matano E, et al. Preventing Venous Thromboembolism in Ambulatory Cancer Patients: the ONKOTEV Study. Oncologist. 2017 May;22(5):601-8.

8 Pabinger I, van Es N, Heinze G, Posch F, Riedl J, Reitter EM, et al. A clinical prediction model for cancer-associated venous thromboembolism: a development and validation study in two independent prospective cohorts. Lancet Haematol. 2018 Jul;5(7):e28998.

9 Khorana AA, Francis CW, Culakova E, Lyman GH. Risk factors for chemotherapy-associated venous thromboembolism in a prospective observational study. Cancer. 2005 Dec;104(12):2822-9.

10 Ay C, Simanek R, Vormittag R, Dunkler D, Alguel G, Koder S, et al. High plasma levels of soluble P-selectin are predictive of venous thromboembolism in cancer patients: results from the Vienna Cancer and Thrombosis Study (CATS). Blood. 2008 Oct;112(7): 2703-8.

11 Ay C, Vormittag R, Dunkler D, Simanek R, Chiriac AL, Drach J, et al. D-dimer and prothrombin fragment $1+2$ predict venous thromboembolism in patients with cancer: results from the Vienna Cancer and Thrombosis Study. J Clin Oncol. 2009 Sep;27(25): 4124-9.

12 Harrison P, Goodall AH. "Message in the platelet"-more than just vestigial mRNA! Platelets. 2008 Sep;19(6):395-404.

13 McBane RD 2nd, Gonzalez C, Hodge DO, Wysokinski WE. Propensity for young reticulated platelet recruitment into arterial thrombi. J Thromb Thrombolysis. 2014;37(2):14854.

14 Lakkis N, Dokainish H, Abuzahra M, Tsyboulev V, Jorgensen J, De Leon AP, et al. Reticulated platelets in acute coronary syndrome: a marker of platelet activity. J Am Coll Cardiol. 2004 Nov;44(10):2091-3. 
15 Nakamura T, Uchiyama S, Yamazaki M, Okubo K, Takakuwa Y, Iwata M. Flow cytometric analysis of reticulated platelets in patients with ischemic stroke. Thromb Res. 2002 May; 106(4-5):171-7.

16 Cesari F, Marcucci R, Gori AM, Caporale R Fanelli A, Casola G, et al. Reticulated platelets predict cardiovascular death in acute coronary syndrome patients. Insights from the AMI-Florence 2 Study. Thromb Haemost. 2013 May;109(5):846-53.

17 Kissova J, Bulikova A, Ovesna P, Bourkova L, Penka M. Increased mean platelet volume and immature platelet fraction as potential predictors of thrombotic complications in BCR/ ABL-negative myeloproliferative neoplasms. Int J Hematol. 2014 Nov; 100(5):429-36.

18 Vasse M, Masure A, Lenormand B. Mean platelet volume is highly correlated to platelet count. Thromb Res. 2012 Sep;130(3):559-60.

19 Thompson CB, Eaton KA, Princiotta SM, Rushin CA, Valeri CR. Size dependent platelet subpopulations: relationship of platelet volume to ultrastructure, enzymatic activity, and function. Br J Haematol. 1982 Mar;50(3): 509-19.

20 Giles H, Smith RE, Martin JF. Platelet glycoprotein IIb-IIIa and size are increased in acute myocardial infarction. Eur J Clin Invest. 1994 Jan;24(1):69-72.

21 Jakubowski JA, Thompson CB, Vaillancourt R, Valeri CR, Deykin D. Arachidonic acid metabolism by platelets of differing size. Br J Haematol. 1983 Mar;53(3):503-11.

22 Bath P, Algert C, Chapman N, Neal B; PROGRESS Collaborative Group. Association of mean platelet volume with risk of stroke among 3134 individuals with history of cerebrovascular disease. Stroke. 2004 Mar;35(3): 622-6.
23 Özlü MF, Öztürk S, Ayhan SS, Tosun M, Alçelik A, Erdem A, et al. Predictive value of mean platelet volume in young patients with nonST-segment elevation acute coronary syndromes: a retrospective observational study. Anadolu Kardiyol Derg. 2013 Feb;13(1):5761.

24 Slavka G, Perkmann T, Haslacher H, Greisenegger S, Marsik C, Wagner OF, et al. Mean platelet volume may represent a predictive parameter for overall vascular mortality and ischemic heart disease. Arterioscler Thromb Vasc Biol. 2011 May;31(5):1215-8.

25 Braekkan SK, Mathiesen EB, Njølstad I, Wilsgaard T, Størmer J, Hansen JB. Mean platelet volume is a risk factor for venous thromboembolism: the Tromsø Study, Tromsø, Norway. J Thromb Haemost. 2010 Jan;8(1):157-62.

26 Riedl J, Kaider A, Reitter EM, Marosi C, Jäger $\mathrm{U}$, Schwarzinger I, et al. Association of mean platelet volume with risk of venous thromboembolism and mortality in patients with cancer. Results from the Vienna Cancer and Thrombosis Study (CATS). Thromb Haemost. 2014 Apr;111(4):670-8.

27 Falanga A, Marchetti M. Hemostatic biomarkers in cancer progression. Thromb Res. 2018 Apr;164 Suppl 1:S54-61.

28 Tinholt M, Viken MK, Dahm AE, Vollan HK, Sahlberg KK, Garred O, et al. Increased coagulation activity and genetic polymorphisms in the F5, F10 and EPCR genes are associated with breast cancer: a case-control study. BMC Cancer. 2014 Nov;14(1):845

29 Ay C, Dunkler D, Simanek R, Thaler J, Koder S, Marosi C, et al. Prediction of venous thromboembolism in patients with cancer by measuring thrombin generation: results from the Vienna Cancer and Thrombosis Study. J Clin Oncol. 2011 May;29(15):2099-103.
30 Reitter EM, Kaider A, Ay C, Quehenberger P, Marosi C, Zielinski C, et al. Longitudinal analysis of hemostasis biomarkers in cancer patients during antitumor treatment. J Thromb Haemost. 2016 Feb;14(2):294-305.

31 Rotteveel RC, Roozendaal KJ, Weijers RN, Eijsman L. Influence of heparin, protamine and polybrene on the time integral of thrombin generation (endogenous thrombin potential). Haemostasis. 1996 Jan-Feb;26(1):1-10.

32 Fine JP, Gray RJ. A Proportional Hazards Model for the Subdistribution of a Competing Risk. J Am Stat Assoc. 1999;94(446):496-509.

33 Choudhury JB. Non-parametric confidence interval estimation for competing risks analysis: application to contraceptive data. Stat Med. 2002 Apr;21(8):1129-44.

34 Bowles KM, Cooke LJ, Richards EM, Baglin TP. Platelet size has diagnostic predictive value in patients with thrombocytopenia. Clin Lab Haematol. 2005 Dec;27(6):370-3.

35 Jeon HK, Kim DU, Baek DH, Ha DW, Lee BE Ryu DY, et al. Venous thromboembolism in patients with cholangiocarcinoma: focus on risk factors and impact on survival. Eur J Gastroenterol Hepatol. 2012 Apr;24(4):444-9.

36 Khorana AA, Francis CW, Culakova E, Kuderer NM, Lyman GH. Frequency, risk factors, and trends for venous thromboembolism among hospitalized cancer patients. Cancer. 2007 Nov;110(10):2339-46.

37 Moore RA, Adel N, Riedel E, Bhutani M, Feldman DR, Tabbara NE, et al. High incidence of thromboembolic events in patients treated with cisplatin-based chemotherapy: a large retrospective analysis. J Clin Oncol. 2011 Sep; 29(25):3466-73.

38 Lee YG, Lee E, Kim I, Lee KW, Kim TM, Lee $\mathrm{SH}$, et al. Cisplatin-Based Chemotherapy Is a Strong Risk Factor for Thromboembolic Events in Small-Cell Lung Cancer. Cancer Res Treat. 2015 Oct;47(4):670-5. 